\title{
Depressed-Cladding 3-D Waveguide Arrays Fabricated With Femtosecond Laser Pulses
}

\author{
Gabriel R. Castillo, Lucía Labrador-Páez, Feng Chen, Senior Member, OSA, Santiago Camacho-López, \\ and Javier R. Vázquez de Aldana
}

\begin{abstract}
We report on the fabrication of waveguide arrays based on depressed-cladding structures produced by femtosecond laser direct inscription in a Nd:YAG crystal. The arrays consisted of seven divergent hexagonal waveguides with different separations between the waveguides at the exit of the crystal. Optical characterization in the visible $(633 \mathrm{~nm})$ and near infrared $(800 \mathrm{~nm})$ was performed. The obtained modal profiles show multimodal behavior in the visible and nearly single-mode at $800 \mathrm{~nm}$. The propagation losses were measured to be $1.6-3.0 \mathrm{~dB} / \mathrm{cm}$ in all the cases. Microluminescence maps were obtained showing the presence of defects only at the damage tracks and a residual stress that slightly affects the waveguide core in the vicinity of the tracks. This affected area is responsible for a modal profile distortion that can be clearly seen at $633 \mathrm{~nm}$, but does not modify the modal profiles in the near infrared. Our results show a very good performance of the fabricated structures and suggest a promising potential use in photonic applications (i.e., photonic lanterns) that can be easily implemented in other transparent materials.
\end{abstract}

Index Terms-Laser materials processing, integrated optics devices, waveguides channeled.

\section{INTRODUCTION}

$\mathbf{F}$ EMTOSECOND laser direct irradiation has consolidated as one of the most versatile and efficient techniques for the inscription of optical waveguides in transparent dielectrics [1]-[5]. Strongly focused femtosecond pulses produce localized modifications in the focal volume inside the material that may consist of very stable (or permanent) refractive-index changes.

Manuscript received January 10, 2017; revised March 15, 2017; accepted April 10, 2017. Date of publication April 23, 2017; date of current version May 15,2017 . This work was supported in part by Junta de Castilla y León (Projects UIC016, SA046U16), MINECO (FIS2013-44174-P, FIS2015-71933-REDT), and in part the Spanish Ministerio de Educación y Ciencia (MAT2013-47395C4-1-R and MAT2016-75362-C3-1-R). The work of L. Labrador-Páez was supported in part by the Universidad Autónoma de Madrid for the "Formación de Personal Investigador (FPI-UAM)" program. (Corresponding author: Gabriel R. Castillo.)

G. R. Castillo and J. R. Vázquez de Aldana are with the Laser Applications and Photonics Group, University of Salamanca, Salamanca 37008, Spain (e-mail: grcastillo@usal.es; jrval@usal.es).

L. Labrador-Paéz is with the Fluorescence Imaging Group, Departamento de Fisica de Materiales, Facultad de Ciencias, Universidad Autonoma de Madrid, Madrid 28049, Spain (e-mail: lucia.labrador@uam.es).

F. Chen is with the School of Physics, Shandong University, Jinan 250100, Shandong, China (e-mail:drfchen@sdu.edu.cn).

S. Camacho-López is with the Departamento de Óptica, División de Física Aplicada, Centro de Investigación Científica y de Educación Superior de Ensenada, Carretera Ensenada-Tijuana No. 3018, C.P. 22860, Ensenada, B.C., Mexico (e-mail: camachol@ cicese.mx).

Color versions of one or more of the figures in this paper are available online at http://ieeexplore.ieee.org.

Digital Object Identifier 10.1109/JLT.2017.2696163
Such modifications can be induced in large areas of the material in a controlled way by properly scanning the sample, thus producing channels in which light can be confined [6]-[8].

In general, depending on the material and on the irradiation conditions (pulse energy, repetition rate, numerical aperture, etc.) different types of material modifications can be induced that can be classified in Type I or Type II modifications [9]. Type I modification is produced when working below the optical damage threshold, and can include either positive or negative refractive index change at the focal volume. Type II modification is produced for pulse energies above the optical damage threshold, and typically consist of a refractive index decrease at the damage track (focal volume) and a refractive index increase at the neighborhood due to the stress field (lattice compression) created during the irradiation [10]. Both types of modifications can be used for fabricating efficient waveguides inside dielectrics. The most straightforward technique consists of finding conditions to produce a refractive index increase with Type I modification, and then scan the sample with the laser focus, once or several times, just to inscribe a channel that directly guides light through. This strategy has been extensively used in glasses, such as fused silica or phosphate glass [1]. However, this approach cannot be successfully implemented in all the cases because there are many materials in which a refractive index increase based on Type I modification has not been achieved. This is the case of many crystalline materials [3] due to the difficulty to produce local densifications in lattice structures, and only in a few of them (such as $\mathrm{LiNbO}, \mathrm{ZnSe}$ and $\mathrm{Nd}: \mathrm{YCa}_{4} \mathrm{O}\left(\mathrm{BO}_{3}\right)_{3}$ ) have been observed Type I modification. Therefore, the strategies for waveguide fabrication in crystals with femtosecond lasers are more frequently based on Type II modifications. Depressed-cladding waveguides [11] are the most versatile structures that can be inscribed with Type II modification, and consist of a large number of parallel damage tracks that form a cladding (with a decreased refractive index), and a central core whose refractive index remains almost unaffected by the femtosecond laser irradiation. Efficient cladding waveguides have been demonstrated in a large number of crystals for different applications (i.e. waveguide lasers or frequency converters) [12]-[17].

In this work we report on the fabrication of optical waveguide arrays with a femtosecond laser, based on depressed-cladding structures. The arrays consist of seven adjacent hexagonal waveguides at the input face, that separate radially in $3 \mathrm{D}$ a certain distance at the output face. We have used a Nd:YAG 


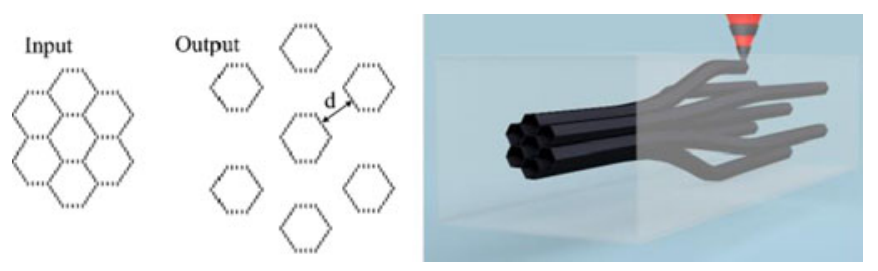

Fig. 1. Schematic diagram of the fabrication of hexagonal waveguide arrays based on depressed-cladding structures produced by femtosecond laser direct inscription.

crystal as host to inscribe the waveguides provided that its spectroscopic properties are very well known, and can be used to map the damage and stress fields at the structures. The modal behavior of the arrays is optically characterized in the visible and near infrared. Microluminescence maps are also presented, showing minimal damage at the waveguide cores. The main interest of the fabricated structures is the potential application in 3D photonic devices, for instance as efficient photonic lanterns. Although our work was focused on Nd:YAG crystal, we have also tested the applicability of our design in other crystals as quartz and $\mathrm{LiNbO}_{3}$.

\section{EXPERIMENTAL SETUP}

\section{A. Waveguide Fabrication}

The femtosecond laser system used for the waveguide inscription (Spitfire, Spectra Physics) emitted 120-fs pulses at a central wavelength of $800 \mathrm{~nm}$ with a $1 \mathrm{kHz}$ repetition rate. A half-wave plate followed by a linear polarizer, and a calibrated neutral density filter, were used to finely tune the pulse energy. The beam was focused through a 50X (numerical aperture 0.6) microscope objective. The sample, a $1 \mathrm{~cm}$ long neodymium doped yttrium aluminum garnet (Nd:YAG) crystal, was placed in a 3-axes motorized stage with a resolution of $0.1 \mu \mathrm{m}$. The threshold energy to produce a damage track at a depth of $200 \mu \mathrm{m}$ from the surface was found to be $0.19 \mu \mathrm{J}$ under our experimental conditions. In order to inscribe the structures, we have used a pulse energy of $0.28 \mu \mathrm{J}$, large enough to ensure the successful inscription of damage tracks at all the required depths of the sample (between 150 and $330 \mu \mathrm{m}$ ), but keeping the damage of the material in a reasonable level such that cracks are not produced. The sample was scanned with the stage at a constant velocity of $500 \mu \mathrm{m} / \mathrm{s}$.

The arrays consisted of seven adjacent hexagonal waveguides, each one formed by 30 parallel tracks with a lateral separation of $2 \mu \mathrm{m}$. This small separation was chosen in order to minimize the light leakage through the cladding boundaries. These parameters produced a single hexagonal structure with a diameter of about $22 \mu \mathrm{m}$. With the aim of demonstrating their ability to be used as photonic lanterns, the design is as follows. The central waveguide is straight from the input to the output faces, and the adjacent 6 waveguides are composed by three parts: a $2-\mathrm{mm}$ straight part (all the waveguides parallel to the central one), a $6 \mathrm{~mm}$ straight segment that separates each waveguide radially from the center, and a final $2 \mathrm{~mm}$ straight part again in the same direction as the initial part (see Fig. 1). Three arrays were

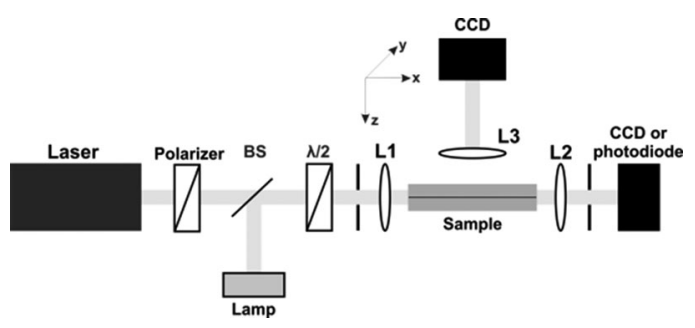

Fig. 2. Characterization setup for the fabricated hexagonal waveguide arrays. Beam splitter (BS) is also used for monitoring of the input beam on the input face of the waveguides.

inscribed with different final separations between the waveguides: WA0 with no separation between waveguides (straight array), WA1 with a separation of $\mathrm{d}=20 \mu \mathrm{m}$, and WA2 with a separation of $\mathrm{d}=40 \mu \mathrm{m}$.

\section{B. Optical Waveguide Characterization}

The ability of the fs-laser written structures to behave as an optical waveguide was investigated with an end-fire coupling setup at $633 \mathrm{~nm}$, at $800 \mathrm{~nm}$ and with white light from a phosphorbased light emitting diode (LED), focused at the input face of the crystal through a $10 \mathrm{X}$ microscope objective. The near-field modal profiles were recorded by imaging the output face of the waveguides through a 20X microscope objective onto a CCD camera as it is shown in Fig. 2. The transmittance measurements were conducted using a calibrated photodiode. In this case, an iris was placed in front of the detector in order to suppress light transmitted through the bulk outside of the waveguide. For this purpose, the size of the iris was fixed by imaging the output face of the waveguides on the CCD and matching the diameter of the iris to that of every single hexagonal structure. Polarization dependence of the waveguides was carried out employing halfwave plates to control the polarization of incident laser beam. Waveguide propagation losses were computed by measuring the input and output powers, taking into account the transmittance of the microscope objectives and Fresnel reflections at the crystal interfaces. In order to obtain a further characterization of losses, the scattered light imaging method was employed, which considers that scattering centers are uniform along the waveguide, where light is propagated [18], [19]. To this end, the coupling of light into the waveguide was monitored by placing a top view imaging system with which we could measure the power decay at the waveguides (see Fig. 2).

The magnitude of the refractive index contrast $(\Delta n)$ between damage tracks and waveguide core was estimated by experimentally measuring the numerical aperture of a single hexagonal waveguide. To this end, lens L2 was removed from the arrangement and the far-field pattern from the waveguide was recorded on the CCD that was placed at a certain distance $D$ from the output face of the waveguide. Then, by using a trigonometric relationship between the radius of the far-field pattern ( $A$, measured from the CCD image) and the distance $D$, the numerical aperture was estimated from:N.A. $=\sin \theta_{m}=A / \sqrt{A^{2}+D^{2}}$. Assuming a step-index profile, $\Delta n$ was calculated using the 

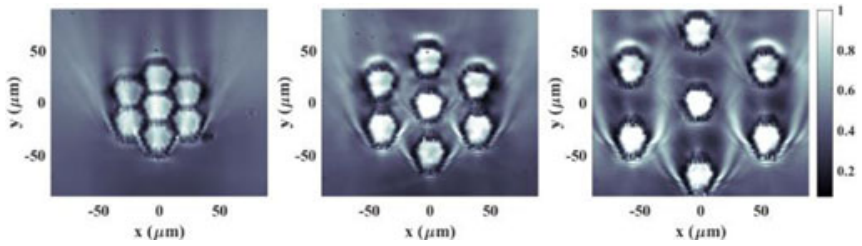

Fig. 3. Optical micrographs of the output face of the waveguide arrays showing confinement for white light for different separations between central and adjacent waveguides (from left to right, WA0, WA1 and WA2). The input faces of the three arrays are identical to the output face of WA0 (picture on the left).

equation [20]

$$
\Delta n \approx \frac{\sin ^{2} \theta_{m}}{2 n},
$$

where $\theta_{\mathrm{m}}$ is the maximum incident angle at which the transmitted power occurs without any change, while $\mathrm{n}$ is the refractive index of the bulk material.

\section{Micro-Luminescence Measurements}

Microluminescence maps of WA2 were obtained using a home-made confocal fluorescence microscope. A single-mode fiber coupled laser diode $(800 \mathrm{~nm})$ was employed as excitation source and focused into the sample by using a microscope objective with 0.85 numerical aperture, leading to an excitation laser spot radius of $0.5 \mu \mathrm{m}$, approximately. $800 \mathrm{~nm}$ radiation excites $\mathrm{Nd}^{3+}$ ions from their ground state $\left({ }^{4} \mathrm{I}_{9 / 2}\right)$ up to their ${ }^{4} \mathrm{~F}_{5 / 2}$ excited state, from which non radiative de-excitations down to the ${ }^{4} \mathrm{~F}_{3 / 2}$ metastable state take place. The subsequent ${ }^{4} \mathrm{~F}_{3 / 2} \rightarrow{ }^{4} \mathrm{I}_{9 / 2}$ emission was collected by the same microscope objective and, after passing through different spatial and spectral filters, was spectrally analyzed by a high-resolution spectrometer. In this work, the attention has been paid to the spatial variation of the intensity, spectral position, and bandwidth of the narrow emission lines centered at around $940 \mathrm{~nm}$, as they have been demonstrated in the past to be excellent indicators of the local damage, stress, and disorder in the Nd:YAG environment, respectively [21]. For the acquisition of fluorescence images of the waveguides, the Nd:YAG sample was scanned in two dimensions by placing it on an XYZ piezo-stage while keeping the $800 \mathrm{~nm}$ excitation spot fixed. The spatial variation of peak intensity, spectral position, and width were obtained by using LabSpec software. The graphical representation of data was performed using the WSxM software [22].

\section{RESULTS AND DISCUSSION}

Firstly, in order to investigate the wave-guiding properties of the written structures, coupling of white light was performed. Fig. 3 shows an optical micrograph of the arrays with different separations between central and adjacent waveguides. As it can be clearly seen, the waveguides present a good confinement of incoherent white light. Furthermore, it is possible to observe that there is no damage of the material in the vicinity of the tracks, even in the zone where the waveguides overlap (in the case of WA0) which assures a good optical confinement and minimizes losses. The modal profiles are homogeneous, showing a nearly
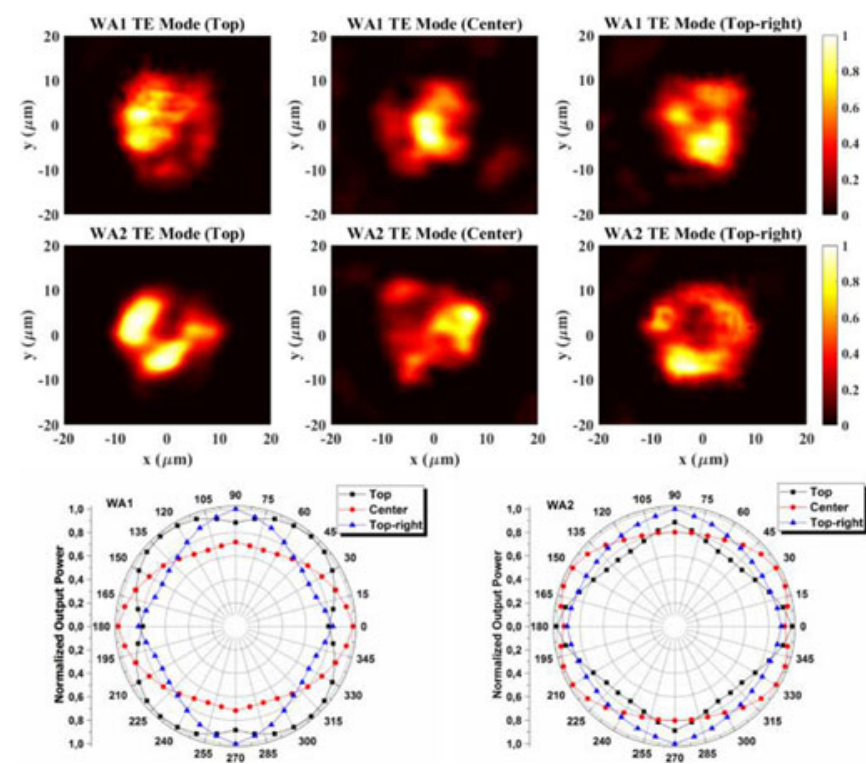

Fig. 4. Modal profiles at $633 \mathrm{~nm}$ for WA1 and WA2 hexagonal waveguides together with their respective maps of polarization dependence, where $0^{\circ}$ corresponds to TE polarization. The dots in the polar maps correspond to the measured power at the output face of the waveguide for every polarization angle, normalized to the maximum output value.

flat-top structure clearly confined by the damage tracks with negligible effect on the separation parameter " $\mathrm{d}$ ".

Furthermore, experiments to characterize the modal behavior of the waveguides at $633 \mathrm{~nm}$ were carried out. Fig. 4 shows the modal profiles for WA1 and WA2 arrays under TE polarization which, in this case, represents polarization perpendicular to the damage tracks. Measurements of every single waveguide in order to observe differences between the central and adjacent waveguides were performed (it should be pointed out that we did not see evidence of coupling of light between adjacent waveguides when only one of them is being excited). For this purpose, images of the modes corresponding to the central and two external waveguides (top and top-right) were taken. It is possible to see in Fig. 4 that there are some differences between modes of adjacent waveguides in comparison with the central one. It can be seen that, at this wavelength, the behavior of the waveguides is multimode, although it is possible to excite only the lower mode in the central waveguide. The other waveguides (external) suffer the bending of the structure and this leads to the excitation of higher order modes. Moreover, it can be seen that in these cases light tends to concentrate near the cladding, maybe due to some residual stress induced during the waveguide fabrication (this effect will be discussed latter, when analyzing the microluminescence maps). Concerning the isotropy of the waveguides (polarization dependence), it was found that waveguides support both TE and TM polarization, with a slight polarization dependence in all of them, being more pronounced in the case of the central ones which, as previously mentioned, could be due to a larger accumulation of mechanical stress induced during the inscription. However, the optical confinement is good in all the cases.

Concerning the propagation losses, measurements for $633 \mathrm{~nm}$ light are summarized in Table I. As it can be seen, the obtained 
TABLE I

Propagation Loses for Central and Adjacent HeXagonal Waveguides

\begin{tabular}{llc}
\hline \hline Waveguide & \multicolumn{2}{c}{$\alpha(\mathrm{dB} / \mathrm{cm}) \pm 0.2$} \\
\hline WA1 & TE & TM \\
Top & 2.7 & 3.0 \\
Center & 3.2 & 2.6 \\
Top-right & 3 & 2.6 \\
WA2 & & \\
Top & & \\
Center & 2.4 & 2.8 \\
Top-right & 2.7 & 2.2 \\
\hline \hline
\end{tabular}

values fluctuate between 1.6 and $3.0 \mathrm{~dB} / \mathrm{cm}$ for the different waveguides and polarizations. The two measurement methods described in Section II were used in some waveguides in order to do a double check of the results. From these measurements some conclusions can be extracted. Firstly, the magnitude of the waveguide losses is similar to those previously reported for other cladding structures in other crystals [12], [15], [23]. Concerning NdYAG, very different values have been reported in previous works, that range from 0.12 [24] to $2.0 \mathrm{~dB} / \mathrm{cm}$ [25], depending on the waveguide diameter and on the coupling wavelength. Due to the small size of the waveguides described in our work ( $22 \mu$ m diameter) and the coupling wavelength used in our studies $(633 \mathrm{~nm})$ our results are in good agreement with [25] (30 $\mu \mathrm{m}$ diameter and same wavelength). Even larger core waveguides [26] may exhibit similar propagation losses values. Secondly, there is no significant difference in the behavior of TE and TM polarizations, as reported in previous works [27]. Finally, the bends of the waveguides have negligible effect in the losses within the accuracy of our measurements. It should be pointed out that the maximum divergence angle of the waveguides is smaller than $0.5^{\circ}$ and this ensures a low level of losses.

Concerning the refractive index contrast induced in the damage tracks, we obtained a value of $\sim 4.2 \times 10^{-3}$ at $633 \mathrm{~nm}$ for one of the straight waveguides using equation (1). It should be stressed that, in this case, this value represents a rough estimation due to the assumption of a step index profile of the waveguides, but that magnitude is in fairly good agreement with previously reported values in Nd:YAG cladding waveguides when large numerical aperture focusing optics was used in the fabrication [28].

The modal characterization at $800 \mathrm{~nm}$ for WA2 is shown in Fig. 5. As it can be seen from the images, the waveguide behavior at this wavelength is nearly single-mode, and distortions of the modal profiles are found only at the external waveguides, due to the effect of the bending. However, the confinement is very good and it was found that the guidance is independent of the incident polarization showing an isotropic behavior in all of the written structures.
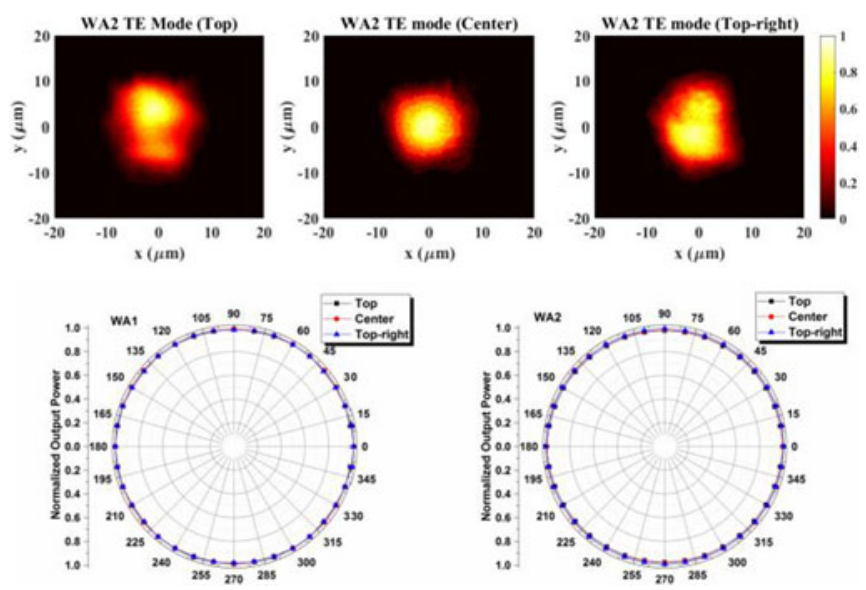

Fig. 5. Modal profiles at $800 \mathrm{~nm}$ for WA2 hexagonal waveguides together with their respective polarization dependence maps. The dots in the polar maps correspond to the measured power at the output face of the waveguide for every polarization angle, normalized to the maximum output value.

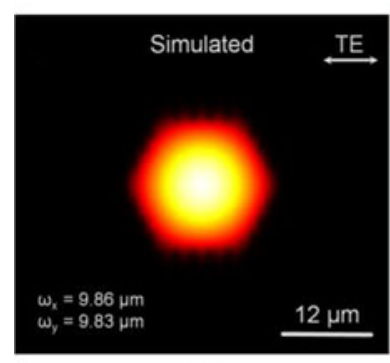

(a)

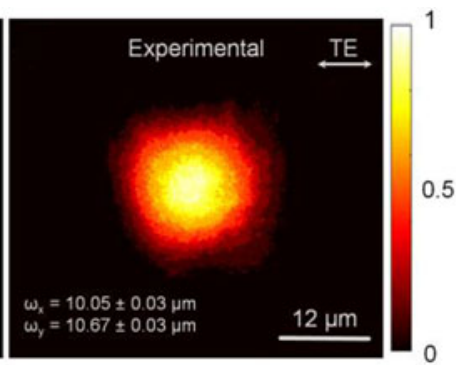

(b)
Fig. 6. (a) Calculated modal profile at $800 \mathrm{~nm}$ of a hexagonal straight waveguide and, (b) measured mode profile at $800 \mathrm{~nm}$ for TE polarization. $\omega_{\mathrm{x}}$ and $\omega_{\mathrm{y}}$ correspond to the mode width sizes, measured at full width at half maximum (FWHM) intensity level, in the horizontal and vertical directions respectively.

Additionally, using the experimentally measured refractive index contrast $(\Delta n)$, a reconstruction of the refractive index profile was carried out. Using this refractive index profile, modal behavior of a straight hexagonal waveguide was calculated by using the finite-difference time-domain method. In Fig. 6, the simulated TE mode at $800 \mathrm{~nm}$ for a straight hexagonal waveguide (neglecting the dependence with wavelength of $\Delta n$ ) together with the experimentally measured mode corresponding to the TE mode center of Fig. 4 are shown. By direct comparing Fig. 6(a) and (b), it is possible to conclude a very good agreement between the simulated and the experimentally measured mode. This indicates that the values used for the reconstruction of the refractive index profile of the waveguide are reasonable.

In order to gain insight in the properties and behavior of the inscribed structures, luminescence maps were taken for the waveguides arrays. Fig. 7 shows the 2D spatial distribution of intensity, induced energy shift, and line width of the ${ }^{4} \mathrm{~F}_{3 / 2} \rightarrow{ }^{4} \mathrm{I}_{9 / 2}$ interStark level emission line of $\mathrm{Nd}^{3+}$ ions around $940 \mathrm{~nm}$ for the input face of the arrays of WA2. In previous works, this emission line has been demonstrated to be an excellent indicative of sensitive changes in the material such as strain, volume changes, 


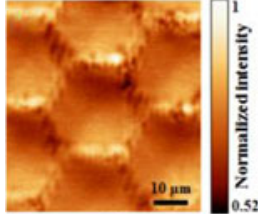

(a)

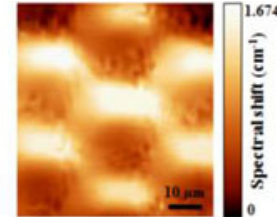

(b)

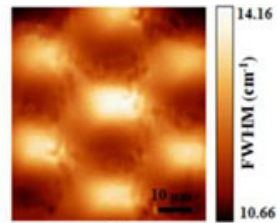

(c)
Fig. 7. Micro-luminescence maps of the input face of the waveguide arrays The images represent the 2D spatial dependence of the intensity (a, peak position (b), and peak width (c) corresponding to the emission line of $\mathrm{Nd}^{3+}$ at $940 \mathrm{~nm}$. maps have shown that the induced damage during the waveguides fabrication is concentrated at the tracks, while a residual stress/strain field affects larger areas of the structures. Our results suggest that the reported waveguide arrays could be used for practical applications in photonics, as for instance in photonic lanterns [29]. Optimization of the modal behavior at the required operation wavelength can be achieved by conveniently adjusting the size of the cladding waveguides.

\section{REFERENCES}

and disorder [21]. The measurements are concentrated mainly in the central area of the array provided it is the most critical region due to the large accumulation of laser impacts of the adjacent waveguides. As it can be seen in Fig. 7(a), there is a clear reduction in the luminescence intensity at the damage tracks, revealing the presence of lattice defects and imperfections in this region. Thanks to the large numerical aperture of the focusing optics employed in the fabrication, the waveguides cores remain almost unaffected. Concerning the spectral position of the emission (Fig. 7(b)) it can be seen an energy redshift (compression) at the damage tracks and under them, and a slight blueshift (dilatation) above them. These effects are related to the stress fields created in the crystal lattice during the irradiation. It should be pointed out that the magnitude of the energy shifts is smaller than the values reported in literature for other cladding structures (see Ref. [27]), however, the extension of the affected areas is larger in our structures. The reason for this effect could be explained in terms of the large density of laser impacts that are used for the fabrication of the waveguide arrays (very small separation between tracks and overlap between claddings of the adjacent waveguides) that lead to a cumulative effect on the stress fields. This inhomogeneous distribution of compressed and dilated areas, could be responsible for the selective concentration of the modal profiles in certain areas of the cladding waveguides (Fig. 3). Fig. 7(c) shows the spectral broadening, that is higher in the zones were more disorder was induced during the fabrication, which correspond with the zones of quenched emission intensity in Fig. 7(a).

\section{CONCLUSION}

We have successfully designed and fabricated 3D arrays of hexagonal cladding waveguides by direct irradiation with femtosecond pulses. The arrays consisted of 7 waveguides that separate radially along the propagation direction. Our study uses a Nd:YAG crystal due to the possibility to microscopically analyze the material modifications induced during the irradiation with the femtosecond laser, but it must be noted that the arrays have been successfully fabricated also in other crystals. The optical characterization of the structure reveals a complex multimodal propagation for the visible, and a nearly single-mode behavior for the NIR; the transmitted power through the waveguides shows a slightly dependence on the incidence laser beam polarization. The measured propagation losses of the waveguides in the arrays are in the range of $1.6-3.0 \mathrm{~dB} / \mathrm{cm}$, with no appreciable effect of the waveguide bends. Micro-luminescence
[1] R. Osellame, G. Cerullo, and R. Ramponi, Femtosecond Laser Micromachining: Photonic and Microfluidic Devices in Transparent Materials. Berlin, Germany: Springer, 2012.

[2] R. R. Gattass and E. Mazur, "Femtosecond laser micromachining in transparent materials," Nature Photon., vol. 2, no. 4, pp. 219-225, Apr. 2008.

[3] F. Chen and J. R. V. de Aldana, "Optical waveguides in crystalline dielectric materials produced by femtosecond-laser micromachining," Laser Photon. Rev., vol. 8, no. 2, pp. 251-275, Mar. 2014.

[4] D. Choudhury, J. R. Macdonald, and A. K. Kar, "Ultrafast laser inscription: Perspectives on future integrated applications," Laser Photon. Rev., vol. 8, no. 6, pp. 827-846, Nov. 2014.

[5] K. Sugioka and Y. Cheng, "Ultrafast lasers-Reliable tools for advanced materials processing," Light Sci. Appl., vol. 3, no. 4, Apr. 2014, Art. no. e149.

[6] K. M. Davis, K. Miura, N. Sugimoto, and K. Hirao, "Writing waveguides in glass with a femtosecond laser," Opt. Lett., vol. 21, no. 21, pp. 1729-1731, Nov. 1996.

[7] K. Miura, J. Qiu, H. Inouye, T. Mitsuyu, and K. Hirao, "Photowritten optical waveguides in various glasses with ultrashort pulse laser," Appl. Phys. Lett., vol. 71, no. 23, pp. 3329-3331, Dec. 1997.

[8] K. Hirao and K. Miura, "Writing waveguides and gratings in silica and related materials by a femtosecond laser," J. Non. Cryst. Solids, vol. 239, no. 1-3, pp. 91-95, Oct. 1998.

[9] S. Gross, M. Dubov, and M. J. Withford, "On the use of the Type I and II scheme for classifying ultrafast laser direct-write photonics," Opt. Express, vol. 23, no. 6, pp. 7767-7770, Mar. 2015.

[10] J. Burghoff, H. Hartung, S. Nolte, and A. Tünnermann, "Structural properties of femtosecond laser-induced modifications in $\mathrm{LiNbO}_{3}$," Appl. Phys. A, vol. 86, no. 2, pp. 165-170, Feb. 2007.

[11] A. G. Okhrimchuk, A. V. Shestakov, I. Khrushchev, and J. Mitchell, "Depressed cladding, buried waveguide laser formed in a YAG:Nd3+ crystal by femtosecond laser writing," Opt. Lett., vol. 30, no. 17, pp. 2248-2250, Sep. 2005.

[12] R. He, Q. An, Y. Jia, G. R. Castillo-Vega, J. R. Vázquez de Aldana, and F. Chen, "Femtosecond laser micromachining of lithium niobate depressed cladding waveguides," Opt. Mater. Express, vol. 3, no. 9, pp. 1378-1384, Sep. 2013.

[13] Y. Jia et al., "Monolithic crystalline cladding microstructures for efficient light guiding and beam manipulation in passive and active regimes," Sci. Rep., vol. 4, Aug. 2014, Art. no. 5988.

[14] W. Nie, Y. Jia, J. R. Vázquez de Aldana, and F. Chen, "Efficient second harmonic generation in 3D nonlinear optical-lattice-like cladding waveguide splitters by femtosecond laser inscription," Sci. Rep., vol. 6, Feb. 2016, Art. no. 22310.

[15] G. Salamu and N. Pavel, "Power scaling from buried depressed-cladding waveguides realized in $\mathrm{Nd}: \mathrm{YVO}_{4}$ by femtosecond-laser beam writing," Opt. Laser Technol., vol. 84, pp. 149-154, Jun. 2016.

[16] H. Liu, Y. Jia, F. Chen, and J. R. Vázquez de Aldana, "Continuous wave duced by inscription of femtosecond laser pulses," Opt. Mater. Express, vol. 3, no. 2, pp. 278-283, Feb. 2013.

[17] Y. Jia, J. R. Vázquez de Aldana, Q. Lu, D. Jaque, and F. Chen, "Second harmonic generation of violet light in femtosecond-laser-inscribed $\mathrm{BiB}_{3} \mathrm{O}_{6}$ cladding waveguides," Opt. Mater. Express, vol. 3, no. 9, p. 1279-1284, Sep. 2013.

[18] F. Wang, F. Liu, G.-K. Chang, and A. Adibi, "Precision measurements for propagation properties of high-definition polymer waveguides by imaging of scattered light," Opt. Eng., vol. 47, no. 2, Feb. 2008, Art. no. O24602. laser operation in Nd:GGG depressed tubular cladding waveguides pro- 
[19] M. H. Jenkins, B. S. Phillips, Y. Zhao, M. R. Holmes, H. Schmidt, and A. R. Hawkins, "Optical characterization of optofluidic waveguides using scattered light imaging," Opt. Commun., vol. 284, no. 16/17, pp. 39803982, Aug. 2011.

[20] J. Siebenmorgen, T. Calmano, K. Petermann, and G. Huber, "Highly efficient Yb:YAG channel waveguide laser written with a femtosecondlaser," Opt. Express, vol. 18, no. 15, pp. 16035-16041, Jul. 2010.

[21] A. Ródenas et al., "Refractive index change mechanisms in femtosecond laser written ceramic Nd: YAG waveguides: Micro-spectroscopy experiments and beam propagation calculations," Appl. Phys. B Lasers Opt., vol. 95, no. 1, pp. 85-96, Jan. 2009.

[22] I. Horcas, R. Fernández, J. M. Gómez-Rodríguez, J. Colchero, J. GómezHerrero, and A. M. Baro, "WSXM: A software for scanning probe microscopy and a tool for nanotechnology," Rev. Sci. Instrum., vol. 78, no. 1, pp. 013705-013708, Jan. 2007.

[23] N. Pavel, G. Salamu, F. Jipa, and M. Zamfirescu, "Diode-laser pumping into the emitting level for efficient lasing of depressed cladding waveguides realized in $\mathrm{Nd}: \mathrm{YVO}_{4}$ by the direct femtosecond-laser writing technique," Opt. Express, vol. 22, no. 19, pp. 23057-23065, Sep. 2014.

[24] A. Okhrimchuk, V. Mezentsev, A. Shestakov, and I. Bennion, "Low loss depressed cladding waveguide inscribed in YAG:Nd single crystal by femtosecond laser pulses," Opt. Express, vol. 20, no. 4, pp. 3832-3843, Feb. 2012.

[25] H. Liu, F. Chen, J. R. Vázquez de Aldana, and D. Jaque, "Femtosecondlaser inscribed double-cladding waveguides in Nd:YAG crystal: A promising prototype for integrated lasers," Opt. Lett., vol. 38, no. 17, pp. 3294 3297, Sep. 2013.

[26] N. Pavel, G. Salamu, F. Voicu, F. Jipa, M. Zamfirescu, and T. Dascalu, "Efficient laser emission in diode-pumped Nd:YAG buried waveguides realized by direct femtosecond-laser writing," Laser Phys. Lett., vol. 10, no. 9 , p. 95802 , Sep. 2013.

[27] H. Liu, Y. Jia, J. R. Vázquez de Aldana, D. Jaque, and F. Chen, "Femtosecond laser inscribed cladding waveguides in Nd: YAG ceramics: Fabrication , fluorescence imaging and laser performance," Opt. Express, vol. 20, no. 17 , pp.-18620-18629, Aug. 2012.

[28] H. Liu, F. Chen, J. R. Vázquez de Aldana, and D. Jaque, "Femtosecondlaser inscribed double-cladding waveguides in Nd:YAG crystal: A promising prototype for integrated lasers," Opt. Lett., vol. 38, no. 17, pp. 3294 3297, Aug. 2013

[29] R. R. Thomson, T. A. Birks, S. G. Leon-Saval, A. K. Kar, and J. BlandHawthorn, "Ultrafast laser inscription of an integrated photonic lantern," Opt. Express, vol. 19, no. 6, pp. 5698-5705, Mar. 2011.

Gabriel R. Castillo received the M.Sc. degree in optics with specialization in optoelectronics from Centro de Investigación Científica y de Educación Superior de Ensenada (CICESE), B.C., Mexico, in 2011. He is currently working toward the Ph.D. degree at the University of Salamanca, Salamanca, Spain. He is a member of the Laser Applications and Photonics Group. His research interest includes ultrafast laser fabrication of photonic devices in transparent dielectrics as well as processing of materials using ultrashort pulsed lasers.
Lucía Labrador-Páez received the degree in physics from the Universidad de La Laguna, Tenerife, Spain, in 2014, the M.Sc. degree in advanced materials and nanotechnology, specializing in photonics, from the Universidad Autónoma de Madrid, Madrid, Spain. She is currently working toward the Ph.D. degree in the Fluorescence Imaging Group, Universidad Autónoma de Madrid. Her research is focused on the spectroscopic properties of nanoparticles dispersed in fluids.

Feng Chen received the Ph.D. degree from Shandong University, Shandong Sheng, China, in 2002. He was with Clausthal University of Technology, Germany, from 2003 to 2005, as an Alexander von Humboldt Research Fellow. He became a Professor at Shandong University in 2006. He is currently a Professor and the Head of School of Physics, Shandong University, China His research interests include material modifications by ultrafast lasers and ion beams, optical waveguides, etc. He is a Fellow of Institute of Physics (IOP) U.K., a senior member of the Optical Society of America (OSA), and Chinese Optical Society (COS). He is also an Associate Editor of Optical Engineering.

Santiago Camacho-López received the B.Sc. and the M.Sc. degrees in physics from Universidad Autonoma Metropolitana-Iztapalapa, Iztapalapa, Mexico, in 1992 and 1994, respectively, and the Ph.D. degree in physics from Imperia College of Science, Technology and Medicine, University of London, U.K., in 2000. From 2000 to 2003, he was a Postdoctoral Fellow in the University of Toronto, Canada. In year 2003, he joined the Department of Optics, CICESE, Ensenada, Baja California, Mexico. Since then, he is a Full-Time Researcher and has chaired the Department (2010-present). His research interest include processing of materials using ultrashort pulse lasers, nonlinear optics, and biophotonics. He holds (2013-present) an Adjunct Professor position in the Mechanical Engineering Department, University of California, Riverside, CA, USA.

Javier R. Vázquez de Aldana received the B.Sc. and Ph.D. degrees from the University of Salamanca, Salmanca, Spain, in 1997 and 2001, respectively. He is currently an Associate Professor of the Science Faculty, University of Salamanca, Salamanca, Spain. His research interests include interaction of intense femtosecond pulses with materials and its application to the fabrication of photonic devices. He is a member of the Laser Applications and Photonics Group, and is also Technical and Scientific Advisor of the Laser Facility, University of Salamanca. 